\title{
Cardiac PET/MR: Are sophisticated attenuation correction techniques necessary for clinical routine assessments?
}

\author{
Martin Lyngby Lassen, PhD, a and Piotr J. Slomka, $\mathrm{PhD}^{\mathrm{a}}$ \\ a Artificial Intelligence in Medicine Program, Cedars-Sinai Medical Center, Los Angeles, CA
}

Received Jan 23, 2020; accepted Jan 24, 2020

doi: 10.1007/s12350-020-02057-9

\section{See related article, pp. 2194-2204}

Cardiac Positron Emission Tomography/Magnetic Resonance Imaging (PET/MR) has gained significant interest since the introduction of the integrated PET/MR systems in 2011. ${ }^{1-3}$ One of the strengths of the hybrid PET/MR systems is the potential for sophisticated cardiorespiratory motion correction of the PET data utilizing well-defined motion vector fields obtained from dedicated MR sequences. ${ }^{4-6}$ In contrast, current applications in PET/CT systems face challenges in the motion correction, which often is based on gated image reconstructions, with high noise and much lower spatial resolution and contrast, when compared to MR images. ${ }^{7}$

The improvements in the image quality and quantitative accuracy obtained from the motion correction in PET/MR systems, however, can be potentially offset by the artifacts in attenuation correction (AC) maps created from MR data (MR-AC maps). In current clinical PET/ MR systems, the MR-AC maps are obtained from either DIXON-VIBE or LAVA-flex sequences. ${ }^{8}$ Both sequences provide in- and opposed phase images, from which fat and water images can be created. Using the fat and water sequences, it is possible to generate MR-AC maps with 2-4 tissue classification (air (background), lung, fat, and soft-tissues, respectively). ${ }^{8}$ Both MR sequences require acquisition times of up to 18 seconds per bed position and, thus, have increased risks of having motion

Reprint requests: Martin Lyngby Lassen, $\mathrm{PhD}$, Artificial Intelligence in Medicine Program, Cedars-Sinai Medical Center, 8700 Beverly Blvd. Ste. A047, Los Angeles, CA 90048; martinlyngby.lassen@cshs.org

J Nucl Cardiol 2021;28:2205-6.

$1071-3581 / \$ 34.00$

Copyright (c) 2020 American Society of Nuclear Cardiology. artifacts (blurring and misalignment) because of respiratory motion during the acquisition. ${ }^{3,8,9}$ These artifacts may translate into streak artifacts in the PET images, misalignment artifacts between the MR-AC maps and the PET emission data, and finally tissue misclassifications. ${ }^{9-12}$ A recent study evaluated the impact of using alternative sequences for the MR-AC maps, with the acquisition protocols based on a multi-echo stack-ofstars gradient echo sequence, which has been proven to be less affected by the cardiorespiratory motion during the acquisition. ${ }^{9}$ While this sequence is a promising alternative to the established MR sequences for MR-AC maps, it is not fully understood how many different tissue classifications are needed to obtain quantitative accurate PET images.

In the current issue of the Journal of Nuclear Cardiology, Robson and colleagues ${ }^{13}$ employed the sequence described by Benkert et al. ${ }^{9}$ with the aim of evaluating how many separate tissues need to be distinguished on MR for the MR-AC map, to obtain PET images of diagnostic quality. The current standard for MR-AC maps (DIXON-VIBE-based AC maps) employs 4- tissue classification. While the use of 4-tissue classification provides sufficient corrections for stationary organs as head and neck, ${ }^{14,15}$ the risk of tissue misclassification artifacts increase with increasing number of segmented tissues. ${ }^{16}$ In their study, Robson and colleagues evaluated a total of four MR-AC maps employing 2 (soft tissue and background), 3 (one including soft tissue, lung, and background - and another including soft tissue, fat, and background), and 4-tissue classification (fat, soft tissue, lung, and background). For evaluation of the respective influence on the PET image reconstructions, the authors evaluated the PET data reconstructed using each of these 4 MR-AC maps both visually (qualitatively), and quantitatively by comparing mean and maximum standardized uptake values (SUV). 
In the study, the authors enrolled nine patients examined for myocardial sarcoidosis using an ${ }^{18} \mathrm{~F}-\mathrm{FDG}$ protocol. From visual assessments of the PET images, the authors were able to conclude that four out of nine patients had differences in the apparent PET tracer uptake when employing MR-AC maps with 2 and 4 tissue classifications, respectively. Nevertheless, despite observing visual differences in the corrected PET images, the authors concluded that all 4 MR-AC maps could be used for PET reconstruction without affecting the clinical assessment. When analyzing the data quantitatively, however, the authors reported differences of magnitude 5-16\% when changing the number of tissue classifications in the MR-AC maps. From the analyses, the authors conclude that the use of two tissue classifications for the MR-AC maps might be sufficient for qualitative assessment of the data, while the use of four tissue classifications is required in studies where quantitative accuracy is required.

The study by Robson et al. ${ }^{13}$ gives rise to an interesting perspective that we should consider for all nuclear cardiology applications using PET/MR systems. Although the visual assessment is sufficient for the identification of myocardial sarcoidosis,${ }^{17}$ the quantitative accuracy might add to the prognostic assessment of the disease burden. In a recent study Ahmadian et al. ${ }^{18}$, the authors found that the use of cardiac metabolic active volume - a semiquantitative assessment evaluating the volume in the heart having uptake value above a background-defined threshold, might provide better prognostic insight than visual assessment alone. Another benefit of using absolute quantification in the assessment of the patients is the potential of automated evaluation of the uptake patterns, reducing visual subjectivity, and bias. Therefore, we should strive to obtain quantitative accurate images rather than settle only for visually comparable images for future applications in PET/ MR as well as PET/CT studies.

\section{References}

1. Delso G, Fürst S, Jakoby B, et al. Performance measurements of the siemens mMR integrated whole-body PET/MR scanner. J Nucl Med 2011;52:1914-22.

2. Nensa F, Bamberg F, Rischpler C, et al. Hybrid cardiac imaging using PET/MRI: A joint position statement by the European Society of Cardiovascular Radiology (ESCR) and the European Association of Nuclear Medicine (EANM). Eur Radiol 2018;2(1):1-16. https://doi.org/10.1007/s00330-017-5008-4.

3. Rischpler C, Nekolla SG, Heusch G, et al. Cardiac PET/MRI-an update. Eur J Hybrid Imaging 2019;3:1-17. https://doi.org/10.118 6/s41824-018-0050-2.

4. Munoz C, Kolbitsch C, Reader AJ, et al. MR-based cardiac and respiratory motion-compensation techniques for PET-MR imaging. PET Clin 2016;11:179-91. https://doi.org/10.1016/j.cpet. 2015.09.004.

5. Munoz C, Neji R, Cruz G, et al. Motion-corrected simultaneous cardiac positron emission tomography and coronary MR angiography with high acquisition efficiency. Magn Reson Med 2017;79:339-50. https://doi.org/10.1002/mrm.26690.

6. Grimm R, Fürst S, Souvatzoglou M, et al. Self-gated MRI motion modeling for respiratory motion compensation in integrated PET/ MRI. Med Image Anal 2014;19:110-20. https://doi.org/10.1016/j. media.2014.08.003.

7. Vandenberghe S, Marsden PK. PET-MRI: A review of challenges and solutions in the development of integrated multimodality imaging. Phys Med Biol 2015;60:R115-54. https://doi.org/10.108 8/0031-9155/60/4/R115.

8. Beyer T, Lassen ML, Boellaard R, et al. Investigating the state-ofthe-art in whole-body MR-based attenuation correction: An intraindividual, inter-system, inventory study on three clinical PET/MR systems. Magn Reson Mater Phys, Biol Med 2016;29:75-87. h ttps://doi.org/10.1007/s10334-015-0505-4.

9. Benkert T, Feng L, Sodickson DK, et al. Free-breathing volumetric fat/water separation by combining radial sampling, compressed sensing, and parallel imaging. Magn Reson Med 2017;78:565-76. https://doi.org/10.1002/mrm.26392.

10. Keller SH, Holm S, Hansen AE, et al. Image artifacts from MRbased attenuation correction in clinical, whole-body PET/MRI. Magn Reson Mater Phys Biol Med 2013;26:173-81. https://doi. org/10.1007/s10334-012-0345-4.

11. Lassen ML, Rasul S, Beitzke D, et al. Assessment of attenuation correction for myocardial PET imaging using combined PET/MRI. J Nucl Cardiol 2017;26:1107-18. https://doi.org/10.1007/s12350017-1118-2.

12. Manber R, Thielemans K, Hutton BF, et al. Practical PET respiratory motion correction in clinical PET/MR. J Nucl Med 2015;56:890-6.

13. Robson PM, Vergani V, Benkert T, et al. Assessing the qualitative and quantitative impacts of simple two-class vs multiple tissueclass MR-based attenuation correction for cardiac PET/MR. J Nucl Cardiol 2020. https://doi.org/10.1007/s12350-019-02002-5.

14. Karakatsanis NA, Abgral R, Trivieri MG, et al. Hybrid PET- and MR-driven attenuation quantification in cardiovascular PET/MR imaging. J Nucl Cardiol 2019. https://doi.org/10.1007/s12350-01901928-0.

15. Ladefoged CN, Law I, Anazodo U, et al. A multi-centre evaluation of eleven clinically feasible brain PET/MRI attenuation correction techniques using a large cohort of patients. Neuroimage 2017; $147: 346-59$

16. Ladefoged $\mathrm{CN}$, Hansen $\mathrm{AE}$, Keller $\mathrm{SH}$, et al. Impact of incorrect tissue classification in Dixon-based MR-AC: Fat-water tissue inversion. EJNMMI Phys 2014;1:101.

17. White JA, Rajchl M, Butler J, et al. Active cardiac sarcoidosis: First clinical experience of simultaneous positron emission tomography-magnetic resonance imaging for the diagnosis of cardiac disease. Circulation 2013;127:e639-41.

18. Ahmadian A, Brogan A, Berman J, et al. Quantitative interpretation of FDG PET/CT with myocardial perfusion imaging increases diagnostic information in the evaluation of cardiac sarcoidosis. $\mathbf{J}$ Nucl Cardiol 2014;21:925-39. https://doi.org/10.1007/s12350-0149901-9.

Publisher's Note Springer Nature remains neutral with regard to jurisdictional claims in published maps and institutional affiliations. 